\title{
Dicke Phase Transition with Multiple Superradiant States in Quantum Chaotic Resonators
}

\author{
C. Liu, ${ }^{1}$ A. Di Falco, ${ }^{2}$ and A. Fratalocchi ${ }^{1, *}$ \\ ${ }^{1}$ PRIMALIGHT, Faculty of Electrical Engineering; Applied Mathematics and Computational Science, \\ King Abdullah University of Science and Technology (KAUST), Thuwal 23955-6900, Saudi Arabia \\ ${ }^{2}$ SUPA, School of Physics and Astronomy, University of St. Andrews, \\ North Haugh, St. Andrews KY16 9SS, United Kingdom \\ (Received 4 February 2014; revised manuscript received 13 April 2014; published 12 June 2014)
}

\begin{abstract}
We experimentally investigate the Dicke phase transition in chaotic optical resonators realized with two-dimensional photonics crystals. This setup circumvents the constraints of the system originally investigated by Dicke and allows a detailed study of the various properties of the superradiant transition. Our experimental results, analytical prediction, and numerical modeling based on random-matrix theory demonstrate that the probability density $P(\Gamma)$ of the resonance widths provides a new criterion to test the occurrence of the Dicke transition.
\end{abstract}

DOI: 10.1103/PhysRevX.4.021048

Superradiance is an emergent property of quantum systems that has stirred a large interest in scientific research [1-6]. Initially predicted by Dicke in the context of twolevel atoms [7], superradiance has been investigated in a wide range of systems including gases [8], plasmas [9], semiconductors [10-12], free-electron lasers [13,14], BoseEinstein condensates [15-19], superconductors [20], quantum systems with impurities [21], and quantum dots $[10,22,23]$. In two-level media, a superradiant state results from the spontaneous synchronization of different atoms immersed in a common radiation field, whose wavelength is larger than the volume occupied by the material. When this condition is met, a quantum phase transition occurs and atoms radiate energy with a quadratic dependence on their population $\left(\propto N^{2}\right)$, much higher than the rate predicted by incoherent spontaneous emission $(\propto N)$ [24-27]. The consequence of such a superradiant behavior is recognized in the spatiotemporal domain, where a directional, shortlived energy burst is generated due to the enhanced radiation rate, while in the case of incoherent emission, only exponentially decaying intensity is observed [2].

The physics of the superradiant phase transition manifests itself in general $\mathrm{N}$-body systems as a self-organization process [4]. In this context, the starting model is that of an effective, non-Hermitian Hamiltonian describing a system with open channels. When the system is closed and the channel strength is 0 , the Hamiltonian is Hermitian and

*andrea.fratalocchi@kaust.edu.sa; http://primalight.org/

Published by the American Physical Society under the terms of the Creative Commons Attribution 3.0 License. Further distribution of this work must maintain attribution to the author(s) and the published article's title, journal citation, and DOI.
Subject Areas: Atomic and Molecular Physics,

Complex Systems,

Interdisciplinary Physics shows real eigenvalues with infinite lifetimes (i.e., zero imaginary component). As the coupling with the environment increases, imaginary eigenvalues appear in the spectrum and resonances become wider in the frequency domain, due to the finite lifetime of the corresponding eigenmodes. When resonances start to overlap, they coherently interact and reorganize, thus originating a phase transition where multiple superradiant states with broad widths emerge in the spectrum [28-30]. The existence of such a transition has also recently been argued as a mechanism to explain the strong deviations from classical Porter-Thomas probability distribution observed in neutron-resonance experiments, thus establishing new connections with the dynamics of complex nuclei [3]. However, if compared to the large body of theoretical results, experimental work has been limited in this area. As a consequence, several properties of superradiant states are still debated, including the emergence of specific scaling laws, the existence of universal statistics, and how these quantities dynamically approach the superradiant transition [31]. Understanding the features of the Dicke transition can be of primary importance not only from a fundamental perspective but also to foster the realization of new devices, including terahertz amplifiers, optical emitters, and laser systems that are under intense investigation [5,6,32-35].

In recent years, due to the many analogies between electrons and photons, light has become a widely used tool to investigate energy-transport dynamics. This analogy is particularly interesting in two dimensions, where the isomorphisms between Schrödinger and Maxwell equations allow us to investigate different quantum phenomena that manifest in dielectric optical microresonators whose forms mimic classically chaotic billiards [36-43]. Here, we show that a suitably engineered optical resonator can mimic 
the dynamics of an open many-body system, allowing for a detailed study of superradiant states. One of the difficulties of the original system investigated by Dicke lies in the requirement of a coupling strength of the order of the energy separation of the atomic energy levels [18]. Our setup, conversely, takes its advantage from the technology of photonics crystals and allows the observation of any coupling regime $[36,44]$. The use of transparent dielectrics, moreover, neglects any unwanted loss mechanism (such as, e.g., material absorption) and provides an ideal platform to investigate different properties of quantum chaotic systems. We begin our analysis by summarizing the main theoretical predictions about superradiance and then present our experimental results with two-dimensional chaotic optical resonators. We then provide a detailed study of the various properties of superradiant states, comparing experimental results with theoretical predictions.

An open quantum $N$-body system can be considered as an effective Hamiltonian $H_{\text {eff }}$ coupled with $M$ decay channels:

$$
H_{\mathrm{eff}}=H_{0}-i \frac{\alpha}{2} V V^{T},
$$

where $H_{0}$ is the Hamiltonian of the closed system and $V$ is an $N \times M$ matrix that models the channel space, with the coupling amplitude defined by $\alpha$. For nonzero $\alpha$, the Hamiltonian $H_{\text {eff }}$ is non-Hermitian and possesses a complex eigenvalue $\Omega_{n}=E_{n}-(i / 2) \Gamma_{n}$ characterized by the energy $E_{n}=\hbar \omega$ and damping (or resonance width) $\Gamma_{n}$. The coupling strength $\kappa$ between the system and the open space (i.e., the continuum of modes) can be evaluated as follows [29]:

$$
\kappa=\frac{\operatorname{Im}\left\{\operatorname{Tr}\left(H_{\mathrm{eff}}\right)\right\}}{N\langle D\rangle}=\left\langle\frac{\Gamma}{D}\right\rangle,
$$

where the $\langle D\rangle$ is the mean energy-level distance and $\langle\Gamma\rangle$ is the mean value of the resonance width $\Gamma$. At low coupling $\kappa \approx 0$, the resonance-width distribution follows a $\chi$-squared distribution [45], while for increasing $\kappa$, appreciable deviations for a $\chi^{2}$ distribution are expected. In the latter case, numerical evidence suggests that the distribution of resonances follows a universal power-law $\Gamma^{-2}$ distribution [3]. The superradiant transition is theoretically predicted in the perfect coupling regime $\kappa=\kappa_{c}=1[3,29,46,47]$, when resonances split and superradiant states emerge in the spectrum. To investigate the appearance of the Dicke transition in our system, we design experiments based on open chaotic cavities realized in two-dimensional photonics crystals (PhCs) in a silicon-on-insulator platform. We chose the $\mathrm{PhC}$ technology for their versatility on managing light behavior on integrated photonics circuits $[48,49]$. Figure 1 shows the SEM image of a typical sample, characterized by a quarter-stadium resonator equipped with input (left channel) and output (right channel) waveguides,

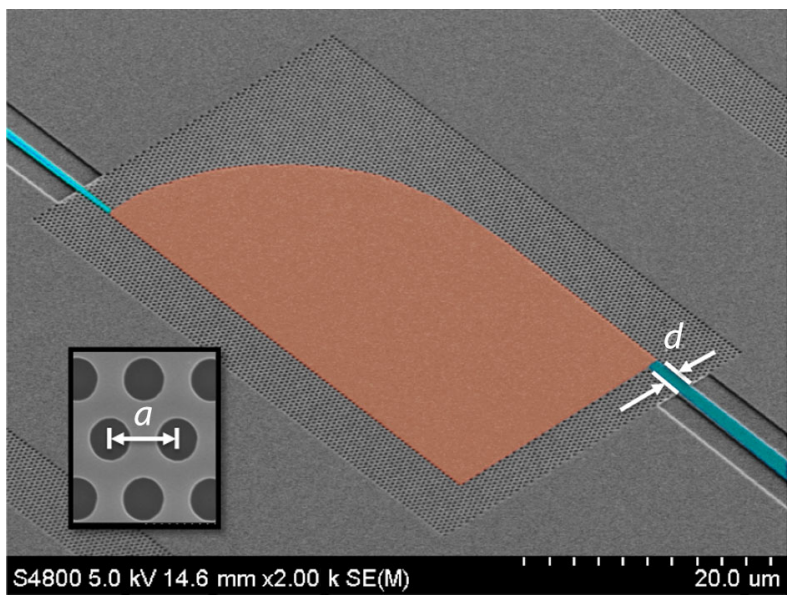

FIG. 1. SEM image of the quarter-stadium resonator. Inset: Enlarged image of the photonic crystal lattice.

the latter with a tunable width $d$. Fabrication details of the structure can be found in Ref. [36]. The stadium shape guarantees that strong chaos is developed in the structure, thus leading to a fully random unperturbed Hamiltonian $H_{0}$. The area of the resonator is $800 \mu \mathrm{m}^{2}$. The inset of the same figure shows an enlarged view of the periodic lattice, designed to work as omnidirectional mirror for light confined and polarized in the plane of the crystal. The period $a=450 \mathrm{~nm}$ and radius $r=0.3 a$ place the working range of wavelength in the $C+L$ band, around $1550 \mathrm{~nm}$. Resonance widths $\Gamma_{i}$ and frequency eigenvalues $\omega_{i}$ can be accurately extracted from the transmitted power-density spectrum measured at the end of the output waveguide, by employing the experimental setup and the wavelet multiscale analysis described, e.g., in Ref. [36]. Figure 2(a) displays a typical experimental spectrum and its reconstruction through multiscale analysis, showing the excellent accuracy of the reconstruction procedure. In order to obtain a complete statistic, we realize 48 samples and collect a total of 7000 resonances.

Figure 2(b) displays the resonance distribution in the plane $(\omega, \Gamma)$ for selected values of output channel width $d$. The latter is measured in lattice unit-cell units and is varied by removing an integer number of rows in the $\mathrm{PhC}$. Figure 2 shows the appearance of a superradiant transition when the spacing increases from $d=1$ to $d=29$. By increasing the waveguide spacing, in fact, we clearly observe resonances splitting with the emergence of a spectral gap [solid area in Fig. 2(b)], dividing the resonance plane $(\omega, \Gamma)$ into two distinct regions: a background containing a large multitude of long-living modes and $M=7$ superradiant states possessing very short lifetimes. The width of such superradiant states is about 100 times larger than the widths measured for $d=1$. In our experiments, the number of modes supported by the cavity is $N \approx 10^{2}$ (as extracted from wavelet multiscale analysis), which shows that the enhancement rate of short-living 

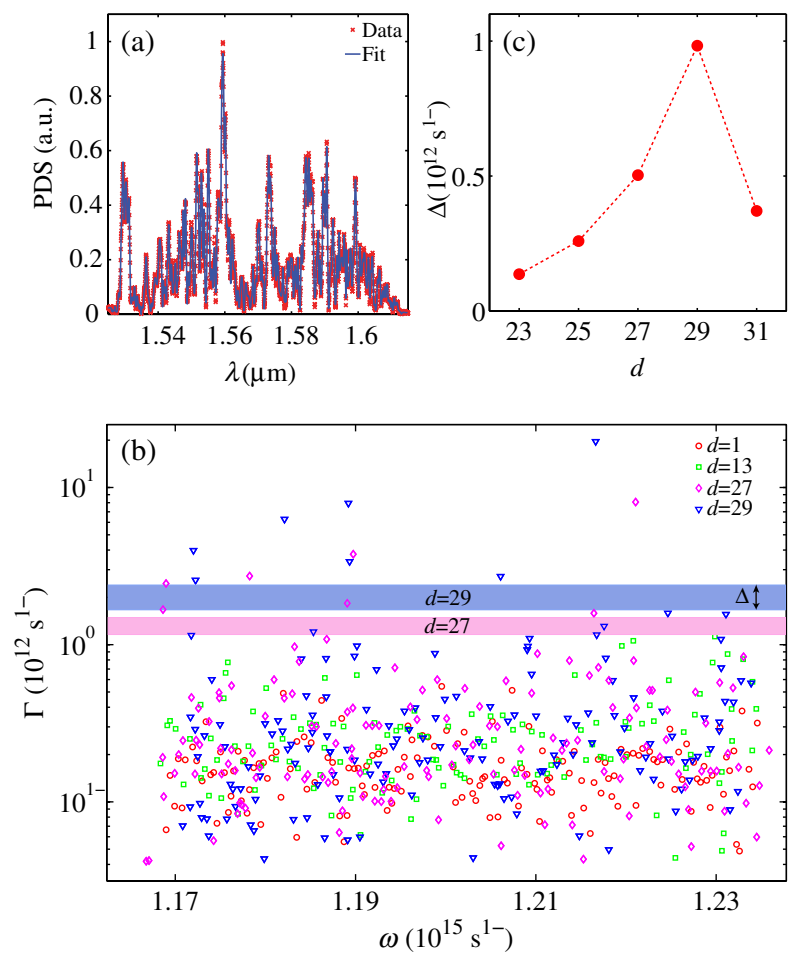

FIG. 2. (a) Experimentally measured power-density spectrum (PDS) (cross markers) and theoretical reconstruction (solid line) via wavelet multiscale analysis for different waveguide spacings $d$. (b) Experimentally extracted resonance $(\omega, \Gamma)$ distribution for different output waveguide spacings $d$. (c) Experimentally measured gap width $\Delta$, which separates superradiant states from long-living resonances, versus $d$.

states is $N$ times larger with respect to noninteracting resonances, in perfect agreement with what is expected for a superradiant behavior. Figure 2(c) shows the behavior of the gap width $\Delta$ versus waveguide spacing $d$. The gap manifests itself for $d \geq 23$ and shows a strongly nonlinear behavior, which is observed as a quadratic increase up to $d=29$ with a further decrease for larger increments of $d$. The specific number $M=7$ of superradiant states observed in our experiments depends on the nature of the cavity losses, which defines the size of the channel space. Multiple waveguide openings with different channel widths $d$, in particular, can sustain the formation of different channel spaces, leading to the generation of different numbers of superradiant states.

To quantitatively validate the occurrence of a superradiant phase transition, we calculate the parameter $\kappa$ of Eq. (2). In order to get a self-consistent evaluation of this parameter, we employ an independent analysis based on random-matrix theory (RMT), which is able to furnish more information-such as, e.g., the number of open channels $M$-about the dynamics of the Dicke transition. To this extent, we begin by diagonalizing an ensemble of $H_{\text {eff }}$ given by Eq. (1) with $H_{0}$ taken from the Gaussian orthogonal ensemble of random matrices and the elements of $V$ obeying a normal distribution with zero mean and unit standard deviation [31]. We then collect large statistics of the random-matrix eigenvalues and calculate the probability distribution $P_{\mathrm{RMT}}(\Gamma)$ of resonance width $\Gamma_{i}$, comparing it with distributions $P_{\exp }(\Gamma)$ calculated from the experimental data of Fig. 2(b). Figure 3 shows typical results in the low coupling [Fig. 3(a)] and superradiant [Fig. 3(b)] regimes. Probability densities $P_{\mathrm{RMT}}(\Gamma)$ are parametrized by the number of open channels $M$ and the coupling strength $\kappa$, the latter varied through $\alpha$, while experimental $P_{\exp }$ distributions depend solely on the losses $d$. In our comparisons, we evaluate parameters $M$ and $\kappa$ by a least-squares fit of the $P_{\mathrm{RMT}}(\Gamma)$ distribution with the corresponding experimental density $P_{\exp }(\Gamma)$ computed at a specific $d$. Quite remarkably, for any spacing $d$, RMT analysis yields a constant channel-space size $M=7$, which perfectly agrees with the experimental results of Fig. 2 that show the appearance of $M=7$ superradiant states. Figures 3(a) and 3(b) also compare experimental results with a $\chi^{2}$ distribution (dashed line). The latter is known to correctly describe the regime of small resonances overlapping, i.e., $\kappa \ll 1$, and well matches the case of $d=1$, while it consistently fails in the superradiant case for $d=29$ due to strong resonances overlapping.

In order to complete our self-consistent evaluation of $\kappa$, we compare RMT predictions with the direct application of Eq. (2) to our experimental results, investigating how the transition is approached when the losses $d$ are increased. Figure 4 shows our results. In general, the value of $\kappa$ calculated through Eq. (2) matches very well the results of RMT, showing the clear appearance of a superradiant phase transition for $d \geq 25$. As the value of $d$ is increased from $d=1$, in particular, the coupling strength $\kappa$ increases from $\kappa \approx 0.3$ and reaches the critical point $\kappa_{c} \approx 1$ at $d=29$. The behavior of $\kappa$ versus $d$ is strongly nonlinear and can be divided into three characteristic regions (Fig. 4). Below $d=25, \kappa$ increases very slowly and linearly with $d$. For $25 \leq d \leq 29$, we observe a dramatic increase toward the critic regime $\kappa_{c}=1$, while for $d \geq 30$, we observe a decrement from $\kappa \approx 1$ to $\kappa \approx 0.7$. The latter is due to the

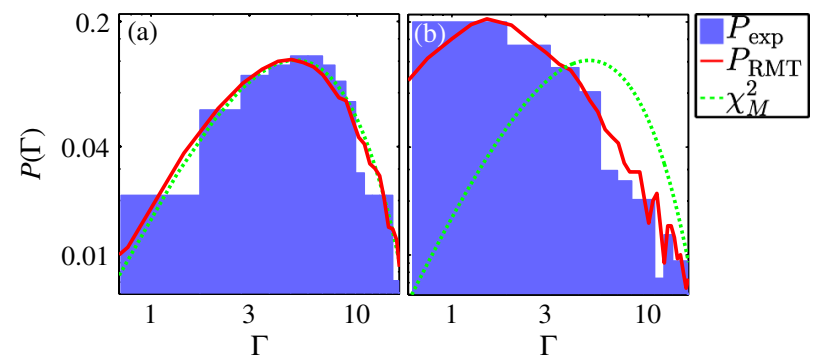

FIG. 3. (a) Experimentally extracted resonance-width distribution $P_{\exp }(\Gamma)$ (solid curve) for (a) $d=1$ and (b) $d=$ 29 , compared with numerically computed densities $P_{\mathrm{RMT}}(\Gamma)$ from random-matrix-theory analysis (solid line) and with a $\chi^{2}$ distribution (dashed line). 


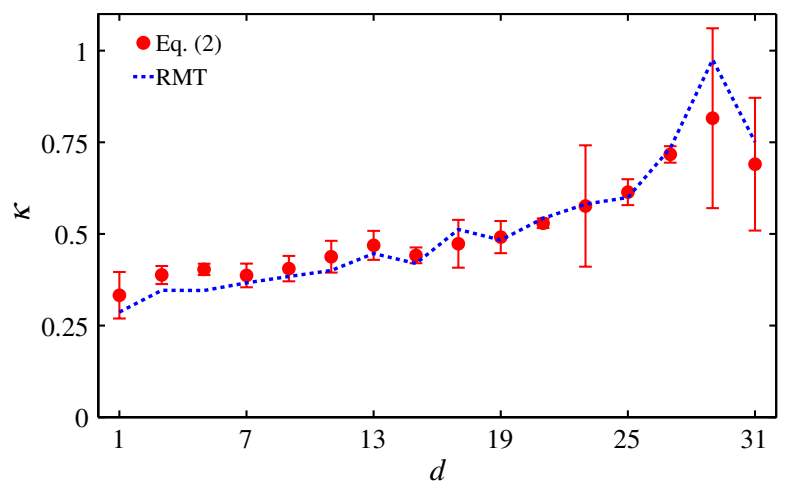

FIG. 4. Experimentally calculated coupling strength $\kappa$ versus $d$ : comparison between estimates from Eq. (2) (markers) and RMT theory (dashed line).

breaking of the chaotic behavior of the resonator when the losses becomes too large, with the consequent weakening of the mixing property of the system.

A further analysis of superradiant transition concerns the scaling law of probability density $P_{\exp }(\Gamma)$ for for large $\Gamma$. There is, in fact, numerical evidence from RMT analysis that the probability density in the superradiant regime follows a universal power law $\propto \Gamma^{-2}$ [3]. If this result is experimentally confirmed, it can provide a new test to verify the presence of a superradiant phase transition. Besides that, our setup also allows us to investigate the transition dynamics and how such power-law distribution is approached. To this extent, we fit the tail of the resonancewidth distribution $P_{\exp }(\Gamma)$ with a power curve $\Gamma^{-\beta}$ and evaluate the coefficient $\beta$ from a least-squares procedure. Figure 5(a) illustrates our results for a varying coupling strength $\kappa$. Figure 5(b) shows a typical outcome of our fitting procedure, displaying an enlarged version of the probability distribution $P(\Gamma)$ for $d=29$. As observed, the probability density at large $\Gamma$ well agrees with a power-law $\Gamma^{-2}$ curve. All the other cases (not reported here) are represented with the same degree of accuracy by a $\Gamma^{-\beta}$
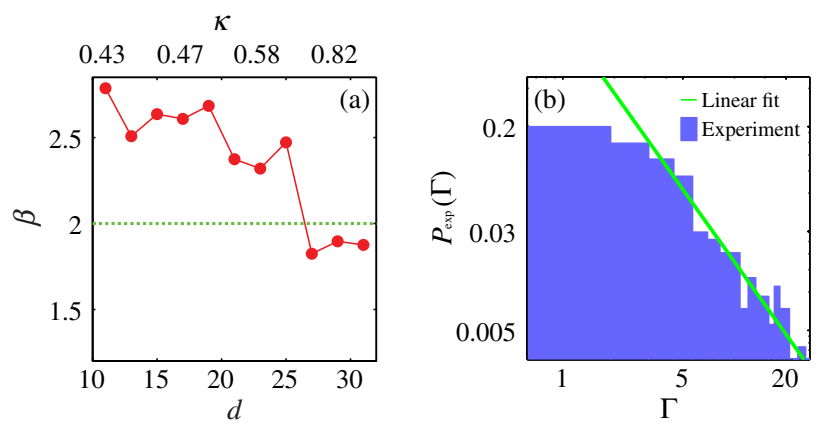

FIG. 5. (a) Power-law exponent $\beta$ (markers) of the large $\Gamma$ behavior of $P_{\exp }(\Gamma) \propto \Gamma^{-\beta}$ at different $d$. The limiting value $\beta=2$ is reported as a dashed line. (b) Comparison between experimental $P_{\exp }(\Gamma)$ for $d=29$ (solid curve) and power-law distribution $\Gamma^{-2}$ (solid line). function. The dynamics of $\beta$ for varying losses $d$ follows a similar behavior of $\kappa$ versus $d$ : For $d<25$, we observe a slowly linear decrease from $\beta \approx 2.5$, while for $d>25$ when the superradiant phase transition appears-the dynamics dramatically converges to $\beta \approx 2$, experimentally confirming the prediction of RMT.

In conclusion, we designed a transparent optical material to investigate the dynamics of the superradiant phase transition in the presence of multiple superradiant states. Our system circumvented the difficulties in observing the Dicke transition in two-level atomic media and allowed a detailed experimental study of superradiant states. Our results showed that the dynamics of the Dicke transition is strongly nonlinear: Characteristic quantities vary slowly below the critical coupling $\kappa_{c}=1$, while near $\kappa_{c}$, the superradiant transition appears dramatically, with the system entering a new self-organized phase. This regime has been experimentally observed by the emergence of $M=7$ superradiant states, whose resonance widths are $N$ times larger than all the others, with $N$ being the total number of resonances. In the superradiant regime, we demonstrated that the resonance-width probability of superradiant states follows a $\Gamma^{-2}$ power law, which provides a new criterion to test the occurrence of a superradiant transition in a physical system. This work is expected to stimulate new fundamental studies on cooperative dynamics and facilitate the development of novel applications of many-body systems.

A.F. acknowledges funding from KAUST (Grant No. CRG-1-2012-FRA-005). A. D. F. acknowledges support from the EPSRC (Fellowships No. EP/I004602/1 and No. EP/J004200/1).

[1] M. Gross and S. Haroche, Superradiance: An Essay on the Theory of Collective Spontaneous Emission, Phys. Rep. 93, 301 (1982).

[2] T. Brandes, Coherent and Collective Quantum Optical Effects in Mesoscopic Systems, Phys. Rep. 408, 315 (2005).

[3] G. L. Celardo, N. Auerbach, F. M. Izrailev, and V. G. Zelevinsky, Distribution of Resonance Widths and Dynamics of Continuum Coupling, Phys. Rev. Lett. 106, 042501 (2011).

[4] K. Hepp and E. H. Lieb, On the Superradiant Phase Transition for Molecules in a Quantized Radiation Field: The Dicke Maser Model, Ann. Phys. (N.Y.) 76, 360 (1973).

[5] D. Meiser, Jun Ye, D. R. Carlson, and M. J. Holland, Prospects for a Millihertz-Linewidth Laser, Phys. Rev. Lett. 102, 163601 (2009).

[6] D. Meiser and M. J. Holland, Steady-State Superradiance with Alkaline-Earth-Metal Atoms, Phys. Rev. A 81, 033847 (2010).

[7] R. H. Dicke, Coherence in Spontaneous Radiation Processes, Phys. Rev. 93, 99 (1954).

[8] N. Skribanowitz, I. P. Herman, J. C. MacGillivray, and M. S. Feld, Observation of Dicke Superradiance in Optically Pumped HF Gas, Phys. Rev. Lett. 30, 309 (1973). 
[9] H. Xia, A. A. Svidzinsky, L. Yuan, C. Lu, S. Suckewer, and M. O. Scully, Observing Superradiant Decay of ExcitedState Helium Atoms inside Helium Plasma, Phys. Rev. Lett. 109, 093604 (2012).

[10] Y. N. Chen, D. S. Chuu, and T. Brandes, Current Detection of Superradiance and Induced Entanglement of Double Quantum Dot Excitons, Phys. Rev. Lett. 90, 166802 (2003).

[11] S.-H. Lim, T. G. Bjorklund, F. C. Spano, and C. J. Bardeen, Exciton Delocalization and Superradiance in Tetracene Thin Films and Nanoaggregates, Phys. Rev. Lett. 92, 107402 (2004).

[12] M. Ichimiya, M. Ashida, H. Yasuda, H. Ishihara, and T. Itoh, Observation of Superradiance by Nonlocal Wave Coupling of Light and Excitons in $\mathrm{CuCl}$ Thin Films, Phys. Rev. Lett. 103, 257401 (2009).

[13] L. Giannessi et al., High-Order-Harmonic Generation and Superradiance in a Seeded Free-Electron Laser, Phys. Rev. Lett. 108, 164801 (2012).

[14] T. Watanabe, X. J. Wang, J. B. Murphy, J. Rose, Y. Shen, T. Tsang, L. Giannessi, P. Musumeci, and S. Reiche, Experimental Characterization of Superradiance in a Single-Pass High-Gain Laser-Seeded Free-Electron Laser Amplifier, Phys. Rev. Lett. 98, 034802 (2007).

[15] S. Inouye, A. P. Chikkatur, D. M. Stamper-Kurn, J. Stenger, D. E. Pritchard, and W. Ketterle, Superradiant Rayleigh Scattering from a Bose-Einstein Condensate, Science 285, 571 (1999).

[16] D. Schneble, Y. Torii, M. Boyd, E. W. Streed, D. E. Pritchard, and W. Ketterle, The Onset of Matter-Wave Amplification in a Superradiant Bose-Einstein Condensate, Science 300, 475 (2003).

[17] S. Inouye, T. Pfau, S. Gupta, A. P. Chikkatur, A. Gorlitz, D. E. Pritchard, and W. Ketterle, Phase-Coherent Amplification of Atomic Matter Waves, Nature (London) 402, 641 (1999).

[18] K. Baumann, C. Guerlin, F. Brennecke, and T. Esslinger, Dicke Quantum Phase Transition with a Superfluid Gas in an Optical Cavity, Nature (London) 464, 1301 (2010).

[19] M. Kozuma, Y. Suzuki, Y. Torii, T. Sugiura, T. Kuga, E. W. Hagley, and L. Deng, Phase-Coherent Amplification of Matter Waves, Science 286, 2309 (1999).

[20] O. Viehmann, J. von Delft, and F. Marquardt, Superradiant Phase Transitions and the Standard Description of Circuit QED, Phys. Rev. Lett. 107, 113602 (2011).

[21] Y.S. Greenberg, C. Merrigan, A. Tayebi, and V. Zelevinsky, Quantum Signal Transmission through a Single-Qubit Chain, Eur. Phys. J. B 86, 368 (2013).

[22] M. Scheibner, T. Schmidt, L. Worschech, A. Forchel, G. Bacher, T. Passow, and D. Hommel, Superradiance of Quantum Dots, Nat. Phys. 3, 106 (2007).

[23] G. Khitrova and H. M. Gibbs, Quantum Dots: Collective Radiance, Nat. Phys. 3, 84 (2007).

[24] N. Lambert, C. Emary, and T. Brandes, Entanglement and the Phase Transition in Single-Mode Superradiance, Phys. Rev. Lett. 92, 073602 (2004).

[25] D. Nagy, G. Kónya, G. Szirmai, and P. Domokos, DickeModel Phase Transition in the Quantum Motion of a BoseEinstein Condensate in an Optical Cavity, Phys. Rev. Lett. 104, 130401 (2010).
[26] V. M. Bastidas, C. Emary, B. Regler, and T. Brandes, Nonequilibrium Quantum Phase Transitions in the Dicke Model, Phys. Rev. Lett. 108, 043003 (2012).

[27] A. Baksic, P. Nataf, and C. Ciuti, Superradiant Phase Transitions with Three-Level Systems, Phys. Rev. A 87, 023813 (2013).

[28] V. V. Sokolov and V. G. Zelevinsky, On a Statistical Theory of Overlapping Resonances, Phys. Lett. B 202, 10 (1988).

[29] V. V. Sokolov and V. G. Zelevinsky, Collective Dynamics of Unstable Quantum States, Ann. Phys. (N.Y.) 216, 323 (1992).

[30] N. Auerbach and V. Zelevinsky, Super-radiant Dynamics, Doorways and Resonances in Nuclei and Other Open Mesoscopic Systems, Rep. Prog. Phys. 74, 106301 (2011).

[31] T. Guhr, A. Müller-Groeling, and H. A. Weidenmüller, Random-Matrix Theories in Quantum Physics: Common Concepts, Phys. Rep. 299, 189 (1998).

[32] M. Asada, Theory of Superradiance from Photon-Assisted Tunneling Electrons and Its Application to Terahertz. Device, J. Appl. Phys. 94, 677 (2003).

[33] T. V. Teperik and A. Degiron, Superradiant Optical Emitters Coupled to an Array of Nanosize Metallic Antennas, Phys. Rev. Lett. 108, 147401 (2012).

[34] J. G. Bohnet, Z. Chen, J. M. Weiner, D. Meiser, M. J. Holland, and J. K. Thompson, A Steady-State Superradiant Laser with Less than One Intracavity Photon, Nature (London) 484, 78 (2012).

[35] O. Kyriienko, A. V. Kavokin, and I. A. Shelykh, Superradiant Terahertz Emission by Dipolaritons, Phys. Rev. Lett. 111, 176401 (2013).

[36] A. Di Falco, T. F. Krauss, and A. Fratalocchi, Lifetime Statistics of Quantum Chaos Studied by a Multiscale Analysis, Appl. Phys. Lett. 100, 184101 (2012).

[37] M. C. Rechtsman, J. M. Zeuner, Y. Plotnik, Y. Lumer, D. Podolsky, F. Dreisow, S. Nolte, M. Segev, and A. Szameit, Photonic Floquet Topological Insulators, Nature (London) 496, 196 (2013).

[38] L. Levi, Y. Krivolapov, S. Fishman, and M. Segev, Hypertransport of Light and Stochastic Acceleration by Evolving Disorder, Nat. Phys. 8, 912 (2012).

[39] T. Pertsch, P. Dannberg, W. Elflein, A. Bräuer, and F. Lederer, Optical Bloch Oscillations in Temperature Tuned Waveguide Arrays, Phys. Rev. Lett. 83, 4752 (1999).

[40] A. Fratalocchi and G. Assanto, All-Optical Landau-Zener Tunneling in Waveguide Arrays, Opt. Express 14, 2021 (2006).

[41] R. Morandotti, U. Peschel, J. S. Aitchison, H. S. Eisenberg, and Y. Silberberg, Experimental Observation of Linear and Nonlinear Optical Bloch Oscillations, Phys. Rev. Lett. 83, 4756 (1999).

[42] H. Trompeter, T. Pertsch, F. Lederer, D. Michaelis, U. Streppel, A. Bräuer, and U. Peschel, Visual Observation of Zener Tunneling, Phys. Rev. Lett. 96, 023901 (2006).

[43] C. Conti and A. Fratalocchi, Dynamic Light Diffusion, Three-Dimensional Anderson Localization and Lasing in Inverted Opals, Nat. Phys. 4, 794 (2008).

[44] C. Liu, A. Di Falco, D. Molinari, Y. Khan, B. S. Ooi, T. F. Krauss, and A. Fratalocchi, Enhanced Energy Storage in Chaotic Optical Resonators, Nat. Photonics 7, 473 (2013). 
[45] C. E. Porter and R. G. Thomas, Fluctuations of Nuclear Reaction Widths, Phys. Rev. 104, 483 (1956).

[46] G. L. Celardo, F. M. Izrailev, V. G. Zelevinsky, and G. P. Berman, Open System of Interacting Fermions: Statistical Properties of Cross Sections and Fluctuations, Phys. Rev. E 76, 031119 (2007).

[47] G. L. Celardo and L. Kaplan, Superradiance Transition in One-Dimensional Nanostructures: An Effective
Non-Hermitian Hamiltonian Formalism, Phys. Rev. B 79, 155108 (2009).

[48] C. Conti, A. Di Falco, and G. Assanto, Optical Parametric Oscillations in Isotropic Photonic Crystals, Opt. Express 12, 823 (2004).

[49] A. Di Falco, C. Conti, and G. Assanto, Impedance Matching in Photonic Crystal Microcavities for Second-Harmonic Generation, Opt. Lett. 31, 250 (2006). 